\title{
Exogenous spermine preserves mitochondrial bioenergetics via regulating Src kinase signaling in the spinal cord
}

\author{
RUI ZHANG，XIN-NAN MA，KAI LIU, LEI ZHANG and MENG YAO \\ Department of Orthopaedics, The Second Affiliated Hospital of Harbin Medical \\ University, Harbin, Heilongjiang 150086, P.R. China
}

Received January 1, 2017; Accepted July 10, 2017

DOI: $10.3892 / \mathrm{mmr} .2017 .7030$

\begin{abstract}
Regulation of mitochondrial metabolism is becoming an important target in inhibiting necrosis and apoptosis following secondary spinal cord injury, and physiological compounds that reduce mitochondrial dysfunction are regarded as efficient protective reagents following injury. It has been demonstrated that spermine, a polyamine composed of four primary amines, may be taken up by a mitochondria-specific uniporter and may preserve mitochondrial bioenergetics, suggesting that it may be important in the pathophysiology of mitochondria. However, the protective mechanism has not yet been definitively clarified. In the present study, isolated spinal cord mitochondria were incubated with spermine to evaluate its physiological functions and Src kinase activities. The results revealed that spermine increased oxidative phosphorylation, attenuated mitochondrial swelling and maintained the membrane potential. An inhibitor of Src kinases, amino-5(4-chlorophenyl)-7-(t-butyl)pyrazolo[3,4-d]pyrimidine (PP2), markedly reduced the effects of spermine. However, inhibition of tyrosine phosphatases by vanadate led to marginal increases in the effects of spermine. Therefore, the present study hypothesized that tyrosine phosphorylation sites are present in the subunits of respiratory chains and mitochondrial permeability transition pore proteins, which may be modified via phosphorylation and dephosphorylation. Furthermore, spermine may upregulate the phosphorylation of Src kinases, and PP2 and vanadate conversely regulate Src phosphorylation. The results of the present study suggest that spermine is a strategic regulator within mitochondria that may activate Src kinases
\end{abstract}

Correspondence to: Professor Meng Yao, Department of Orthopaedics, The Second Affiliated Hospital of Harbin Medical University, 246 Xuefu Road, Harbin, Heilongjiang 150086, P.R. China

E-mail: yaomeng1952@126.com

Abbreviations: RCR, respiratory control ratio; MPTP, mitochondrial permeability transition pore

Key words: spermine, Src kinases, respiratory control ratio, mitochondrial permeability transition pore, spinal cord in the spinal cord, and tyrosine phosphorylation signaling is a primary regulatory pathway of mitochondrial metabolism.

\section{Introduction}

Mitochondria are the powerhouse organelles of mammalian cells, producing $>90 \%$ of adenosine triphosphate (ATP) which is used to maintain cellular functions. However, mitochondria are also known to be major participants in free radical production and calcium $\left(\mathrm{Ca}^{2+}\right)$ buffering. During the pathophysiological process of secondary traumatic spinal cord injury (SCI), dysfunction of oxidative phosphorylation leads to an accumulation of excessive reactive oxygen species (ROS) within the mitochondria $(1,2)$. Meanwhile, the excess $\mathrm{Ca}^{2+}$ ions are transported to the mitochondrial matrix in order to sustain the homeostatic content of $\mathrm{Ca}^{2+}$ within the cytosol $(3,4)$. However, an excess of ROS and $\mathrm{Ca}^{2+}$ induces the opening of mitochondrial permeability transition pores (MPTPs), which non-selectively allow any micromolecule with a molecular weight of $\leq 1.5 \mathrm{kDa}$ to pass through the mitochondrial inner membrane $(5,6)$. The subsequent influx of water attenuates the osmotic pressure of the mitochondrial matrix, leading to mitochondrial swelling. The outward expansion leads to the disappearance of inner membrane cristae and, eventually, rupture of the outer membrane (7). Mitochondrial rupture causes the release of ROS, $\mathrm{Ca}^{2+}$ and pro-apoptotic factors, including the electron carrier cytochrome $c$ (cyt $c$ ), apoptosis-inducing factor (AIF) and others, into the cytosol. Collectively, these substances activate several forms of programmed cell death, including necroptosis, apoptosis and autophagy (8-10). Therefore, maintaining the proper function of oxidative phosphorylation and inhibiting MPTP opening are important therapeutic approaches to alleviate secondary traumatic SCI.

Spermine is a biological organic aliphatic polycation with four primary amino groups. It is ubiquitously present in all living cells, including bacterial, plant and animal cells. In humans, spermine was initially identified in sperm cells, where it is involved in proliferation and differentiation. Naturally occurring spermine is synthesized in the cytosol and then transported to the mitochondrial matrix. A specific spermine uniporter is present in the mitochondrial outer and inner membranes, and has a low affinity and high capacity for spermine storage (11). The spermine, accumulated in the 
uniporter, can be released into the matrix according to a shift in electric transmembrane potential (12). In the mitochondrial matrix, spermine acts on the pyruvate dehydrogenase complex and respiratory chain complexes to regulate oxidative phosphorylation activity (13). Furthermore, it has been demonstrated that spermine is involved in scavenging ROS and inhibiting $\mathrm{Ca}^{2+}$-induced MPTP opening, depending on its concentration in the matrix $(14,15)$. However, the mechanisms by which spermine protects mitochondrial functions are not fully understood.

Accumulating evidence has indicated that tyrosine phosphorylation has profound effects on mitochondrial functions; dozens of proteins distributed in mitochondrial subcomponents are the targeted substrates of tyrosine kinases and phosphatases, including certain subunits of respiratory chain complexes, cyt $c$ and the pyruvate dehydrogenase complex (16). Furthermore, it is increasingly clear that several essential components of the MPTP contain modulation sites of tyrosine phosphorylation; this includes hexokinase type I in the cytosol, voltage-dependent anion channel (VDAC) in the outer membrane, adenine nucleotide translocator (AdNT) in the inner membrane, and subunit $\gamma$ of ATP synthase in the matrix $(17,18)$. Thus, tyrosine kinases and phosphatases can substantially adjust the activity of the MPTP by reversible phosphorylation and dephosphorylation. Src family kinases (SFKs), non-receptor tyrosine kinases that are stably and abundantly present within mitochondria, have been shown to be crucial participants in the process of reducing ROS levels, particularly in rat brain mitochondria (19). In contrast to SFKs, tyrosine phosphatases, such as SHP-2 and PTP1B, attenuate this scavenging function. Notably, spermine and SFKs appear to have similar effects on the regulation of oxidative phosphorylation and inhibition of MPTP opening. Nevertheless, to the best of our knowledge, no definitive evidence has been presented of an association between spermine and SFKs in spinal cord mitochondria.

The present study demonstrates that spermine increases the process of oxidative phosphorylation and inhibits MPTP opening in mitochondria isolated from the spinal cord. These effects are reduced in the presence of an SFK inhibitor and are enhanced by a phosphatase inhibitor. These findings illustrates that spermine activates SFKs, which subsequently maintains mitochondrial bioenergetics.

\section{Materials and methods}

Animals and reagents. Female Sprague-Dawley rats weighing $220 \pm 20$ g were obtained from Experimental Animal Center of Harbin Medical University (Harbin, China). The rats were used in experimental procedures in accordance with the Guide for Care and Use of Laboratory Animals approved by the China National Institutes of Health. Spermine and cyclosporin A (CsA) were purchased from Sigma-Aldrich (Merck Millipore, St. Louis, MO, USA). Amino-5-(4-chlorophenyl)-7-(t-butyl) pyrazolo[3,4- $d$ ]pyrimidine(PP2) was also purchased from Sigma-Aldrich and dissolved in DMSO. Vanadate was obtained from Chemservice SA (Grevenmacher, Luxembourg). The JC-1 fluorescence probe and BCA Protein Assay kit were obtained from Beyotime Institute of Biotechnology (Shanghai, China). Primary antibodies against Src, phospho-Y419, phosphor-Y530 and COX IV were purchased from Abcam (Cambridge, MA,
USA), and the alkaline phosphatase (AP)-linked IgG secondary antibody was purchased from ZSGB Biotech, Co., Ltd. (Beijing, China). Calcium chloride $\left(\mathrm{CaCl}_{2}\right)$ was acquired from Sunshine Biotech, Co., Ltd. (Nanjing, China).

Isolation of spinal cord mitochondria. Rat spinal cord mitochondria were isolated by a conventional differential centrifugation method, as modified from a previously described procedure (20). Briefly, animals were decapitated after anesthetizing with $10 \%$ chloral hydrate $(3 \mathrm{ml} / \mathrm{kg}$, intraperitoneally). The spinal cord segments from T8 to L1 were rapidly dissected and homogenized in isolation buffer $\mathrm{A}$ [320 mM sucrose, $5 \mathrm{mM}$ HEPES, $0.5 \mathrm{mM}$ EDTA, $0.1 \%$ bovine serum albumin (BSA) ( $\mathrm{pH}$ 7.4)] with Dounce tissue grinders. The homogenate was subjected to centrifugation at $1,000 \mathrm{xg}$ for $10 \mathrm{~min}$ and supernatant was then centrifuged at $13,000 \mathrm{xg}$ for $10 \mathrm{~min}$. The mitochondrial pellet was subsequently suspended in isolation buffer B (210 mM mannitol, $70 \mathrm{mM}$ sucrose, $10 \mathrm{mM}$ succinate, $10 \mathrm{mM}$ HEPES, 0.1\% BSA, pH 7.4) for use in later steps. The freshly prepared mitochondria were used within $4 \mathrm{~h}$, and all procedures were performed at $4^{\circ} \mathrm{C}$.

Standard incubation procedures. The mitochondrial suspension was centrifuged prior to incubation. The pellet was resuspended with respiration medium containing $230 \mathrm{mM}$ mannitol, $70 \mathrm{mM}$ sucrose, $5 \mathrm{mM}$ succinate, $3 \mathrm{mM}$ HEPES and $2 \mathrm{mM}$ Tris-phosphate ( $\mathrm{pH}$ 7.4). Spermine (1 mM) was added to the isolated mitochondria (1 $\mathrm{mg}$ of protein), except for the control group. PP2 $(10 \mu \mathrm{M})$ or vanadate $(1 \mathrm{mM})$, inhibitors of SFK or phosphatase, respectively, was then added to regulate tyrosine phosphorylation. Incubation was conducted for $30 \mathrm{~min}$ at $20^{\circ} \mathrm{C}$. Mitochondrial protein concentrations were determined using the BCA Protein Assay kit.

Mitochondrial respiration control ratio $(R C R)$. Mitochondrial RCR was assessed with Oxygraph Clark-Type electrodes (Hansatech Instruments, Norfolk, England) as described previously (21). In a sealed chamber, the incubated mitochondria were added to the respiration buffer $(125 \mathrm{mM} \mathrm{KCl}, 2.5 \mathrm{mM}$ $\mathrm{KH}_{2} \mathrm{PO}_{4}, 2 \mathrm{mM} \mathrm{MgCl}, 20 \mathrm{mM}$ HEPES, $0.1 \% \mathrm{BSA}, \mathrm{pH} 7.2$ ) to yield a final protein concentration of $500 \mu \mathrm{g} / \mathrm{ml}$. Measurement of mitochondrial respiration (oxygen consumption) was initiated by addition of pyruvate $(5 \mathrm{mM})$ and malate $(2.5 \mathrm{mM})$, which are the oxidative substrates of complex I, designated as state II respiration. This was followed by the addition of adenosine diphosphate (ADP; $1 \mathrm{mM}$ ) to induce state III respiration, and then addition of oligomycin $(1 \mu \mathrm{M})$ as state IV respiration. RCR was calculated as the ratio of the slope of the state III respiration curve to the slope of the state IV respiration curve, which is considered to be a sensitive measure of mitochondrial function, indicating the integrity of the inner membrane and the capacity to perform oxidative phosphorylation.

Determination of mitochondrial membrane potential $\left(\Delta \Psi_{m}\right)$. To measure $\Delta \Psi_{\mathrm{m}}$, JC-1, a lipophilic cation-susceptive fluorescent probe, was added to the mitochondria according to manufacturer's instructions. Briefly, $0.9 \mathrm{ml} \mathrm{JC}-1$ dye working solution $(0.2 \mathrm{X})$ was added to $0.1 \mathrm{ml}$ mitochondria $(0.1 \mathrm{mg}$ of protein) at the beginning of standard incubation (as described above). After mixing with other reagents, $0.2 \mathrm{ml}$ suspension 
was added into each well of a 96-well microplate. Fluorescence intensity was instantly measured by time scan using a fluorospectro-photometer (Shimadzu, Kyoto, Japan) with red and green channels. JC-1 aggregates were formed in the presence of a higher $\Delta \Psi_{\mathrm{m}}$, emitting red fluorescence (excitation $525 \mathrm{~nm}$, emission $590 \mathrm{~nm}$ ), whereas monomers were formed in the presence of a lower $\Delta \Psi_{\mathrm{m}}$, indicated by green fluorescence (excitation $490 \mathrm{~nm}$, emission $530 \mathrm{~nm}$ ). The emissions ratio of aggregates to monomers was used to determine $\Delta \Psi_{\mathrm{m}}$ levels. In addition, carbonyl cyanide $m$-chlorophenyl hydrazone (CCCP), an uncoupler of oxidative phosphorylation, was added as positive control for inner membrane depolarization.

$\mathrm{Ca}^{2+}$-induced MPTP opening. MPTP opening was evaluated through $\mathrm{Ca}^{2+}$ overload in vitro, as previously described (22). Following incubation with various reagents, isolated mitochondria were added into a 96-well microplate with $0.2 \mathrm{ml}$ of respiration medium. $\mathrm{CaCl}_{2}(100 \mu \mathrm{M})$ was added to each well in order to abruptly increase the extramitochondrial $\mathrm{Ca}^{2+}$ concentration. As a $\mathrm{Ca}^{2+}$ buffering organelle, excess $\mathrm{Ca}^{2+}$ was taken up by mitochondria, resulting in an elevation of the $\mathrm{Ca}^{2+}$ concentration in the mitochondrial matrix. Accumulated $\mathrm{Ca}^{2+}$ induced MPTP opening, facilitating an influx of $\mathrm{H}_{2} \mathrm{O}$ into the mitochondrial matrix according to the change of osmotic pressure. Water influx led to mitochondrial swelling, accompanied by an increase in volume and attenuation of absorbance at 540 $\mathrm{nm}$; the decreasing degree of absorbance was used as an indicator of the sensitivity of MPTP to $\mathrm{Ca}^{2+}$ overload. The $540 \mathrm{~nm}$ absorbance of the mitochondrial suspension was monitored with a microplate reader (Bio-Rad Laboratories, Inc., Hercules, CA, USA) every two min. CsA $(1 \mu \mathrm{M})$, an inhibitor of MPTP, was used as positive control.

Western blot analysis. Western blot analysis was used to detect the phosphorylation level of residue Y419, the activation site, and residue Y530, the inhibition site of Src kinases. Mitochondria were incubated at $20^{\circ} \mathrm{C}$ with spermine in the presence of PP2 or vanadate and, after $30 \mathrm{~min}$, the reaction was stopped by the addition of lysis buffer containing $50 \mathrm{mM}$ Tris- $\mathrm{HCl}, 150 \mathrm{mM}$ $\mathrm{NaCl}, 2$ mM EDTA, 2 mM EGTA, 0.2\% Triton X-100, 0.3\% NP-40, $1 \mathrm{mM}$ DTT and $100 \mu \mathrm{M}$ phenylmethylsufonyl fluoride. Following incubation on ice for $30 \mathrm{~min}$, the mixtures were centrifuged at $12,000 \mathrm{x}$ g for $15 \mathrm{~min}$ to remove mitochondrial membrane fragments. The protein concentration was also determined by BCA Protein Assay kit. Equal amounts of protein from groups incubated with different agents were loaded and separated by $10 \%$ sodium dodecyl sulfate polyacrylamide gel electrophoresis, and the samples were then transferred to polyvinylidene difluoride membranes using a Semi-Dry Transfer Cell (Bio-Rad Laboratories, Inc.). The membrane was blocked in TBST solution with 5\% skimmed milk at room temperature for $2 \mathrm{~h}$. Subsequently, the membrane was incubated overnight at $4{ }^{\circ} \mathrm{C}$ with primary antibodies against Src (dilution, 1:5,000) and phospho-Y419 (dilution, 1:5,000) and phospho-Y530 (dilution, 1:500) diluted in TBST. Following three washes, the membrane was incubated with the AP-IgG secondary antibody (dilution, 1:5,000) for $1 \mathrm{~h}$ at room temperature. The protein bands were detected using a FluorChem E imaging analysis system and AlphaView software 3.4.0.0 (Cell Biosciences, Inc., Santa Clara, CA, USA).
Src was used as the loading control for semi-quantitative analysis.

Statistical analysis. All data are presented as the mean \pm standard error. Data from RCR and western blotting were analyzed by one-way analysis of variance (ANOVA) among the groups using SPSS 18.0 software package (SPSS, Inc., Chicago, IL, USA). When appropriate, a post-hoc test was performed using least significant differences (LSD) test. Data analysis from the detection of $\mathrm{Ca}^{2+}$-induced MPTP opening and $\Delta \Psi_{\mathrm{m}}$ was performed by a repeated-measures ANOVA and LSD post-hoc test over the experimental period. The differences among the groups were considered significant at $\mathrm{P}<0.05$ (two-tailed).

\section{Results}

Spermine increases the RCR of spinal cord mitochondria. The effects of spermine on mitochondrial respiration (state III, state IV and RCR) were examined following incubation with spermine, PP2 or vanadate, as well as in the control group (Fig. 1), indicating that spermine markedly altered the mitochondrial bioenergetics of oxidative phosphorylation. Typical curves representing mitochondrial respiration in the spermine and control group are shown in Fig. 1A, illustrating the successful isolation of the mitochondria by a procedure with high functional quality. Treatment with spermine led to an apparent increase in the slope of ADP phosphorylation (state III). While the sole PP2, an inhibitor of Src kinases, marginally decreased the slope of state III. Based on the post-hoc analysis of total data, the magnitude of state III respiration following spermine treatment was increased by $16 \%$ compared with the control $(\mathrm{P}<0.05$; Fig. 1C). However, no significant changes were detectable in state IV respiration between the spermine and control groups $(\mathrm{P}>0.05$; Fig. $1 \mathrm{~A}$ and $\mathrm{C}$ ). Consequently, there was a significant increase in the RCR of the spermine group compared with the control $(\mathrm{P}<0.05$; Fig. 1D). Representative respiration curves following the addition of spermine with PP2 are shown in Fig. 1B; in the spermine with PP2 group, state III respiration was observed to be decreased by $40 \%$ compared with the spermine group and by $22 \%$ compared with the control group $(\mathrm{P}<0.05$; Fig. $1 \mathrm{C})$, and state III respiration had no significant difference compared with the sole PP2 group ( $\mathrm{P}>0.05$; Fig. $1 \mathrm{C})$. Due to the absence of significant changes in state IV respiration, the RCR of spermine with PP2 was decreased by $47 \%$ compared with the spermine group $(\mathrm{P}<0.01)$, suggesting that Src kinases were the major regulators of ADP phosphorylation (Fig. 1D). Following the addition of vanadate, the curves of state III respiration, state IV respiration and RCR exhibited similar tendencies to those in the spermine group, as shown in Fig. 1B, which further demonstrated that tyrosine phosphorylation was modulated by spermine.

Spermine maintains $\Delta \Psi_{m}$ independently of tyrosine phosphorylation. In energized mitochondria, aggregates of JC-1 accumulate in the mitochondrial matrix emitting red fluorescence due to the potential difference across the inner membranes. Following membrane depolarization, aggregates dissociate into monomers with green fluorescence. Thus, the fluorescence intensity ratio of aggregates to monomers 


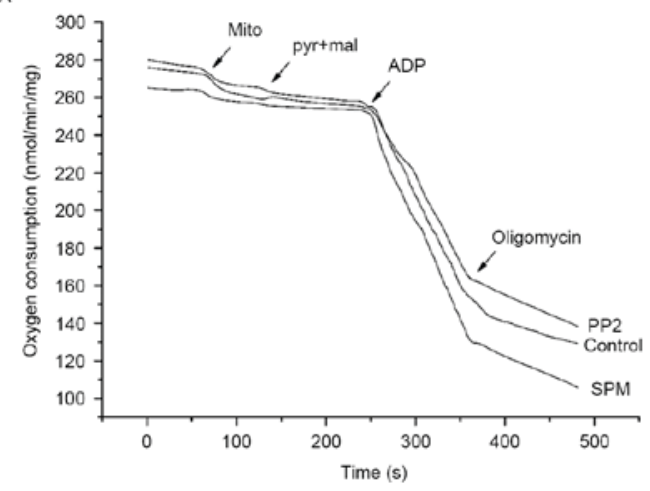

c

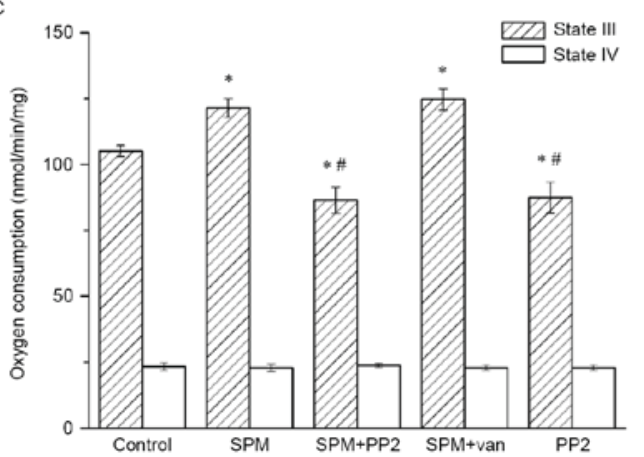

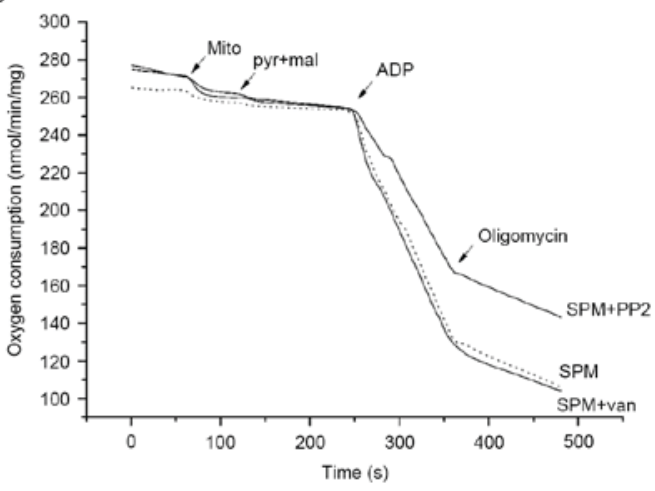

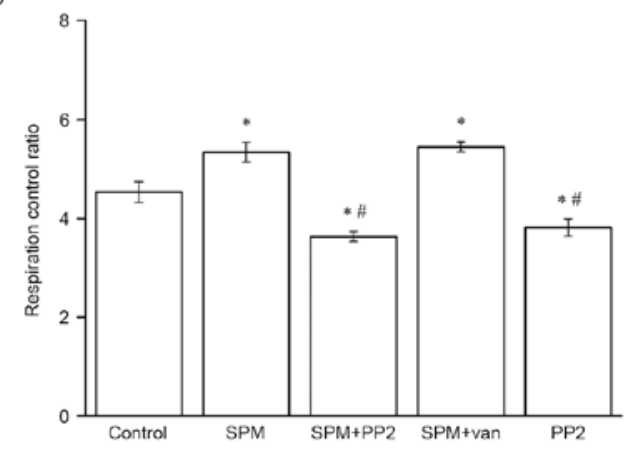

Figure 1. Effects of spermine on mitochondrial oxidative phosphorylation. Isolated mitochondria (500 $\mu \mathrm{g}$ of protein) were incubated in respiration medium including $230 \mathrm{mM}$ mannitol, $70 \mathrm{mM}$ sucrose, $5 \mathrm{mM}$ succinate, $3 \mathrm{mM}$ HEPES and $2 \mathrm{mM}$ Tris-phosphate (pH 7.4) for 30 min before oxygen consumption measurements using Clark-type electrode. (A) Respiration curves of incubated mitochondria. Respiration medium was in the presence of spermine (1 mM) or sole PP2 $(10 \mu \mathrm{M})$. Addition times of mitochondria, pyruvate $(5 \mathrm{mM})$ and malate $(2.5 \mathrm{mM})$, ADP $(1 \mathrm{mM})$, oligomycin $(1 \mu \mathrm{M}$, an inhibitor of ATP Synthase) were labeled in the figure by arrows. (B) Respiration curves of mitochondria with spermine and inhibitors. Mitochondria were subjected to spermine addition PP2 $(10 \mu \mathrm{M})$ or vanadate $(1 \mathrm{mM})$. The slopes represented the velocities of oxygen consumption. (C) Oxygen consumption of state III and state IV respiration. State III respiration was induced by addition of ADP and state IV respiration was began with oligomycin. To the state III respiration, spermine significantly increased the oxygen consumption compared with the control, whereas this effect was partially inhibited by sole or additional PP2. However, there was no significant difference between the groups of state IV respiration. (D) Mitochondrial RCR. The RCR is the slopes ratio of state III to state IV which reflected the mitochondrial bioenergetics and oxidative phosphorylation. The RCR was significantly increased by addition of spermine compared with the control, however, sole or additional PP2 both attenuated the RCR compared with the spermine group. SPM spermine, van vanadate, mito mitochondria, pyr pyruvate, mal malate. Results shown are mean $\pm \mathrm{SD}$ with $\mathrm{n}=6$ for each group. ${ }^{*} \mathrm{P}<0.05$ vs. control group; ${ }^{*} \mathrm{P}<0.05$ vs. spermine group. $\mathrm{PP} 2$, amino-5(4-chlorophenyl)-7-(t-butyl)pyrazolo[3,4-d]pyrimidine; ADP, adenosine diphosphate; ATP, adenosine triphosphate; RCR, respiratory control ratio.

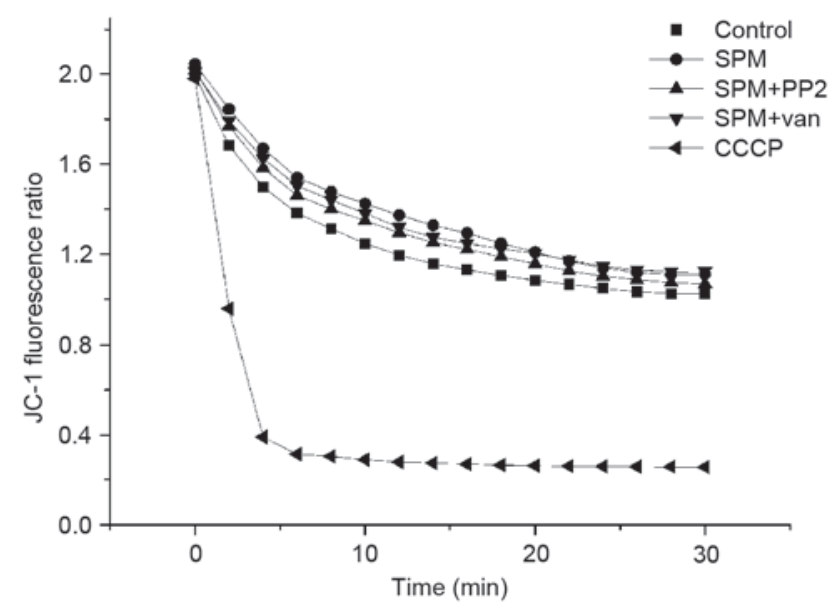

Figure 2. Determination of mitochondrial membrane potential $\left(\Delta \Psi_{\mathrm{m}}\right)$ on JC-1 staining. Mitochondria were incubated in the same conditions as shown in Fig. 1. JC-1 dyeing working solution (0.2x) was present for $\Delta \Psi_{\mathrm{m}}$ measurements. $\Delta \Psi_{\mathrm{m}}$ was determined by the emissions ratio of aggregates to monomers $(590 \mathrm{~nm} / 530 \mathrm{~nm})$. CCCP $(10 \mu \mathrm{M})$ was used to entirely depolarize the membrane potential as positive control. SPM spermine, van vanadate. Each plot is representative of six independent measurements. CCCP, carbonyl cyanide m-chlorophenyl hydrazone. reflects variations in $\Delta \Psi_{\mathrm{m}}$. As shown in Fig. 2, the addition of spermine marginally increased the $\Delta \Psi_{\mathrm{m}}$ ratio compared with the control group $(\mathrm{P}<0.05)$. However, this observation opposed results previously obtained in liver mitochondria, in which the positive charges of the mitochondria neutralized the negative potential in the matrix, leading to a decrease in $\Delta \Psi_{\mathrm{m}}(23)$. The different findings may be explained by the fatty acid contents of membrane phospholipids; as abundant fatty acids within the central nervous system mitochondrial membranes in the spinal cord have a physiologically high proton permeability, which respond rapidly to a slight change of $\Delta \Psi_{\mathrm{m}}(19)$. In the presence of positive charges, spermine partially neutralized the anionic groups of membrane phospholipids, resulting in an increase of $\Delta \Psi_{\mathrm{m}}$ value. Also, in this case, $\Delta \Psi_{\mathrm{m}}$ was not influenced by the addition of PP2 or vanadate compared with the spermine group ( $\mathrm{P}>0.05)$. The uncoupling reagent $\mathrm{CCCP}$ (used as a positive control) caused an instant dissipation of $\Delta \Psi_{\mathrm{m}}$ due to the opening of the membrane proton leak channel.

Spermine inhibits MPTP opening in isolated spinal cord mitochondria. A reduction in absorbance may be observed in response to the swelling of mitochondria, reflecting the 

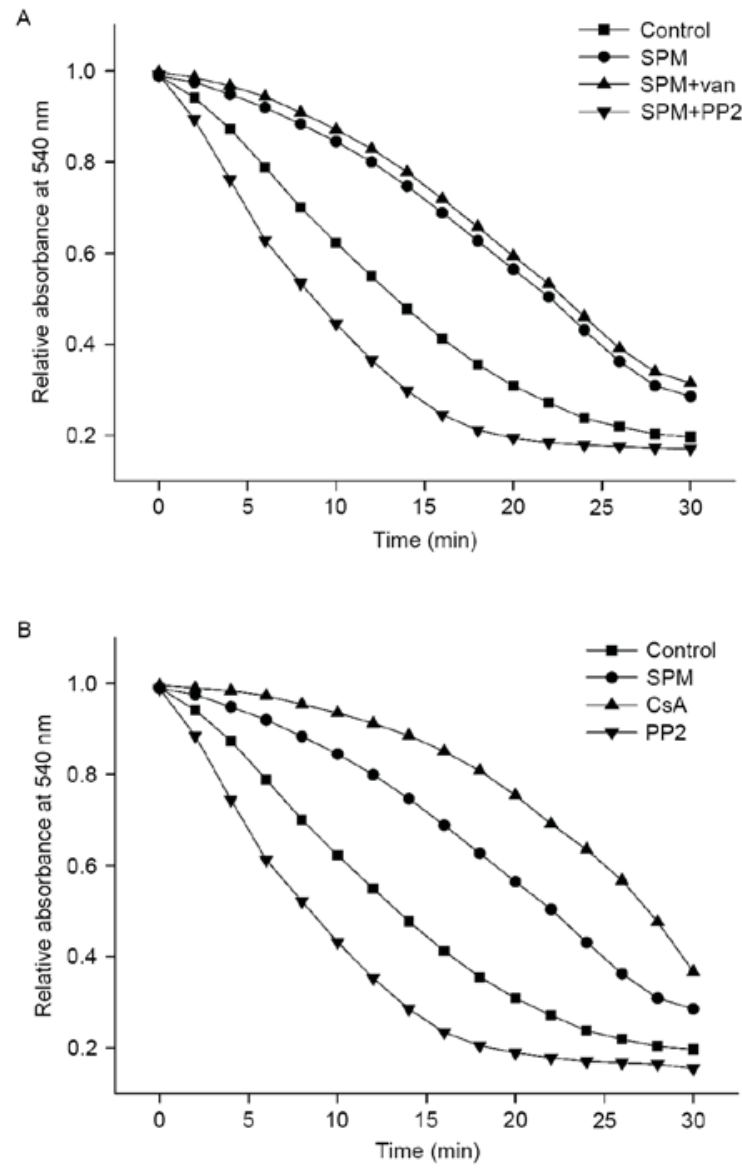

Figure 3. Effects of spermine on $\mathrm{Ca}^{2+}$-induced MPTP opening. Mitochondria (1 $\mathrm{mg}$ of protein) were incubated in respiration medium as Fig. 1 described with spermine $(1 \mathrm{mM})$ except the control, sole PP2 and CsA curves. (A) The MPTP opening curves of mitochondria with spermine and inhibitors. PP2 $(10 \mu \mathrm{M})$ or vanadate $(1 \mathrm{mM})$, inhibitor of SFK or phosphatase, was added to spermine for regulation tyrosine phosphorylation. (B) The MPTP opening curves of mitochondria with control reagents. CsA $(1 \mu \mathrm{M})$ was used to prevent the MPTP opening as positive control. PP2 $(10 \mu \mathrm{M})$ without spermine was used to inhibit tyrosine phosphorylation. Absorbance at $540 \mathrm{~nm}$ was monitored in the presence of $\mathrm{CaCl}_{2}(100 \mu \mathrm{M})$. SPM spermine, van vanadate, CsA cyclosporine A. Each plot is representative of six independent measurements. MPTP, mitochondrial permeability transition pores; PP2, amino-5-(4-chlorophenyl)-7-(t-butyl)pyrazolo[3,4-d]pyrimidine; CsA, cyclosporin A; SFK, Src family kinases.

opening of ion channels and membrane pores. To clarify whether the $\mathrm{Ca}^{2+}$-activated channels or membrane pores were MPTPs, CsA, a specific inhibitor of MPTP, was added to the buffer solution as positive control. The change in relative absorbance at $540 \mathrm{~nm}\left(\Delta \mathrm{A}_{540}\right)$ over the initial $20 \mathrm{~min}$ was assessed to evaluate the functions of spermine. As shown in Fig. $3 \mathrm{~A}, \mathrm{CaCl}_{2}$ induced rapid swelling of energized mitochondria in the standard respiration medium; however, this effect was greatly inhibited by CsA, indicating that MPTPs were only $\mathrm{Ca}^{2+}$-activated channel or pores. In the presence of spermine $(1 \mathrm{mM}), \Delta \mathrm{A}_{540}$ was attenuated by $40 \%$ compared with the control group $(\mathrm{P}<0.01)$, suggesting that spermine may incompletely inhibit MPTP opening.

To identify the regulatory effect of spermine on tyrosine phosphorylation, PP2 $(10 \mu \mathrm{M})$, with or without spermine, was added into the respiration medium, both resulting in accelerated swelling of mitochondria and significantly increased rate of $\Delta \mathrm{A}_{540}$. The curves of spermine with PP2 group and sole PP2 had an approximate tendency, and the relative absorbance both decreased sharply, reaching its plateau after $\sim 14 \mathrm{~min}$, which was a shorter time interval than that in other group. These findings implied that tyrosine dephosphorylation may increase the sensitivity of MPTP opening. As tyrosine phosphorylation is regulated by kinases and phosphatases, vanadate $(1 \mathrm{mM})$, an inhibitor of phosphatases, was used to enhance phosphorylation; this slightly attenuated the $\Delta \mathrm{A}_{540}$ compared with the spermine group, indicating that vanadate and spermine had homologous effects on the regulation of MPTP opening.

Spermine upregulates the phosphorylation levels of Src kinases within mitochondria. The protein expression levels of Src kinases were evaluated by immunoblot analysis (Fig. 4). This illustrated that spermine treatment was associated with a significant upregulation of activated Src kinase (phospho-Y419) compared with the control group $(\mathrm{P}<0.05)$. Compared with the spermine-treated mitochondria, the addition of PP2 significantly downregulated the expression level of phospho-Y419 $(\mathrm{P}<0.01)$. Notably, vanadate exhibited a parallel function: reducing the phosphorylation of residues Y419, as observed for PP2. This may be related to the modification of Src kinase activation; the process of activation is initiated by the dephosphorylation of inhibitory residues Y530, which is performed by tyrosine phosphatases. As an inhibitor of phosphatases, vanadate prevented the activation of residues Y419.

To ascertain whether CsA induced tyrosine phosphorylation of the MPTP subunits, CsA was individually added into the mitochondria and western blot analysis was subsequently performed. The results showed that there was no significant difference compared with the control group $(\mathrm{P}>0.05)$. These findings suggest that spermine may be a crucial regulator of Src kinases.

\section{Discussion}

The present study provides evidence that exogenous spermine significantly increases oxidative phosphorylation activity and inhibits the opening of MPTP in mitochondria isolated from the spinal cord. Furthermore, SFKs play an essential role in modulating these physiological processes, and are dependent on reversible phosphorylation and dephosphorylation. These data suggest that spermine potently preserves mitochondrial bioenergetics by regulating the activities of SFKs.

Mitochondrial dysfunction can induce a series of pathophysiological alterations, including a deficiency of ATP synthesis, increase of ROS production and release of pro-apoptotic factors, which further cause the necrosis and apoptosis of cells, as well as edema and inflammation within tissues. These process are ubiquitous in hepatic injury, myocardial ischemia reperfusion, and particularly evident in secondary SCI (2). Several previous studies reported that spermine could improve the mitochondrial functions in various tissue, whereas the underlying mechanisms were quite different between central nervous system and other organs $(24,25)$. In brain tissue, the transport process of spermine into mitochondria were regulated by tyrosine phosphorylation, which was differ from liver mitochondria regulation by peroxides (19). In the present study, it was confirmed that spermine induces an increase of RCR 

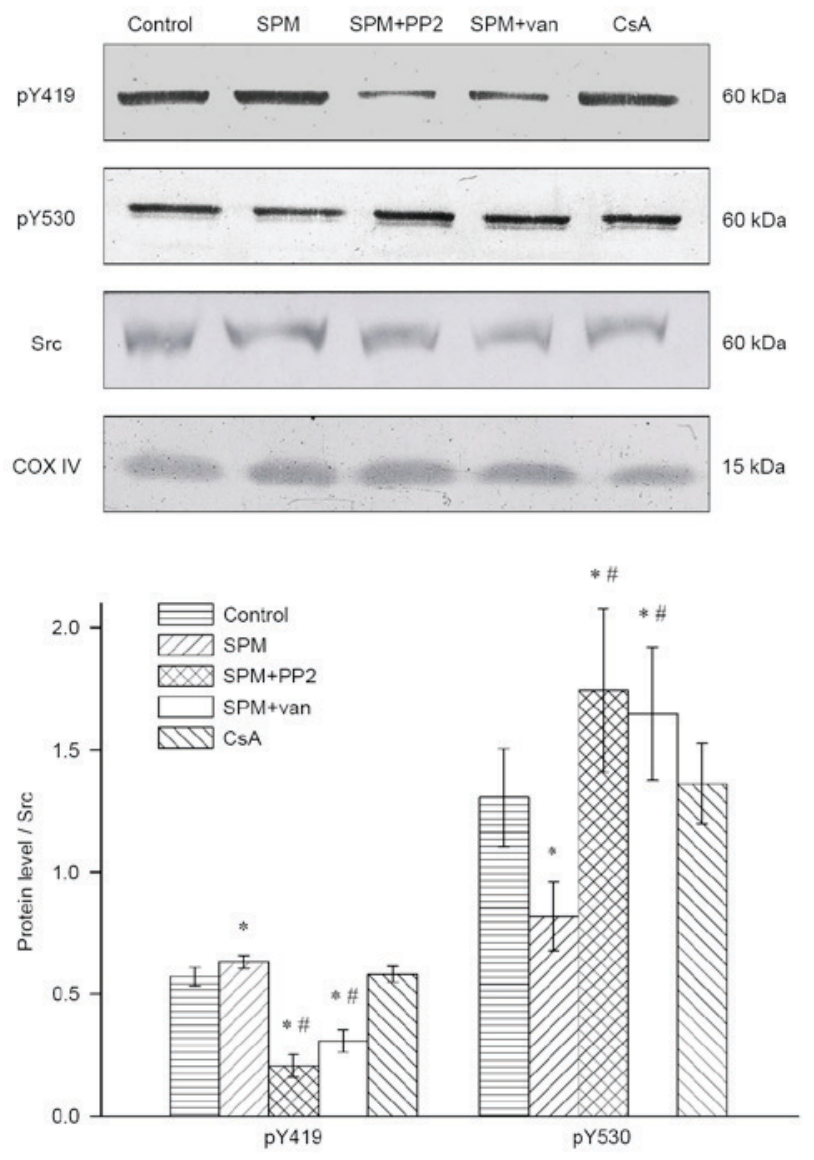

Figure 4. Spermine increased the phosphorylation of Src kinases in the isolated mitochondria. The top panel showed representative protein expression levels of phosphor-Y419/Y530 in the immunoblot analysis. Src reported for loading control. The bottom panel represented the ratio of interest proteins immunoreactivity to that of Src for each group. SPM spermine, van vanadate, CsA. Results shown are mean $\pm \mathrm{SD}$ with $\mathrm{n}=6$ for each group. " $\mathrm{P}<0.05$ vs. control group and ${ }^{\#} \mathrm{P}<0.01$ vs. spermine group. CsA, cyclosporin $\mathrm{A}$.

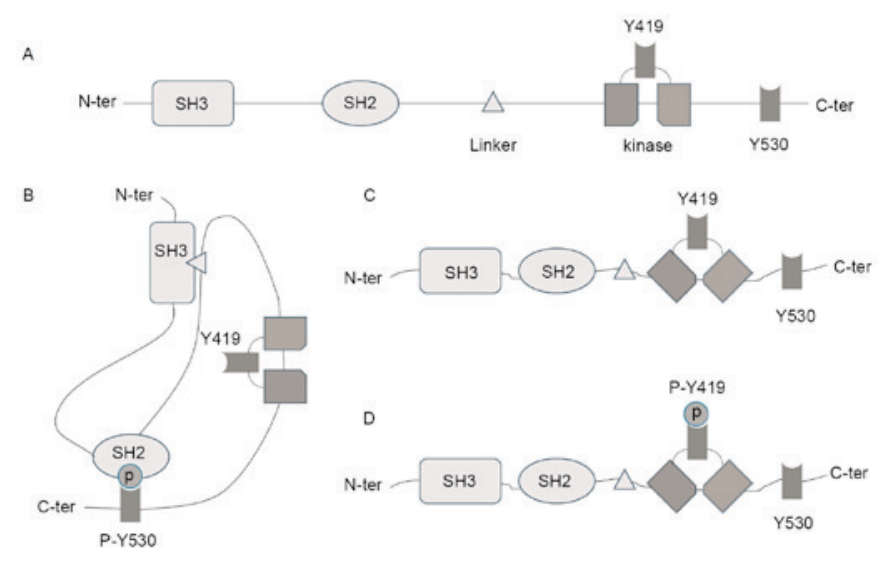

Figure 5. Structure and activation process of Src kinases. (A) Different members of the Src kinases family have the common domains from N- to C-terminal. SH3 domain is the binding site of linker. SH2 domain interacts with phospho-Y530 residue. Y419 residue is the activation site of kinase domain. (B) Inactive Src kinases structure: phospho-Y530 residue bind to the SH2 domain, and the linker with proline-rich sequence combines to the SH3 domain. The dual connections crimp the molecule to a close loop structure which masks the activation site Y419. (C) Intermediate state of the kinases in activating process: dephosphorylation of Y530 triggers the opening of the molecular structure. Then the kinase domain is further modified to expose the Y419 residue. (D) Active Src kinases structure: phosphorylation of Y419 residue activates the Src kinases completely.

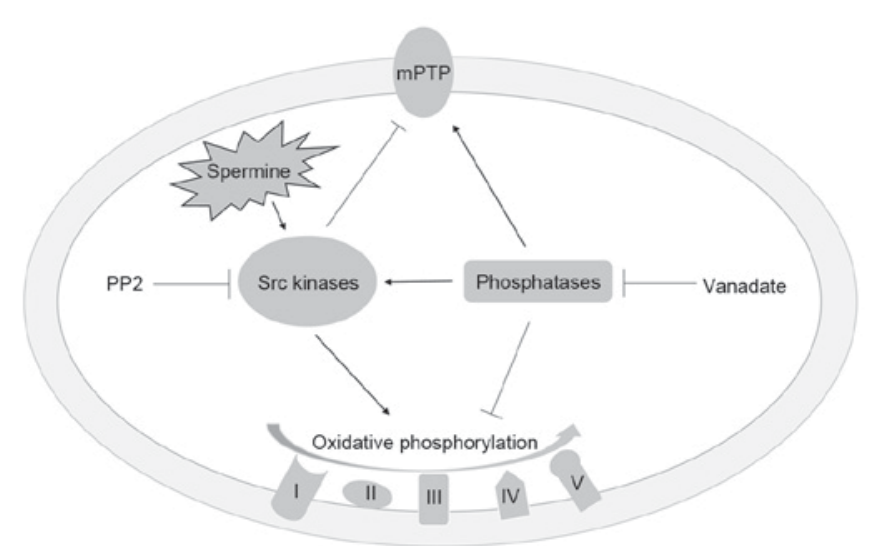

Figure 6. Effects of Src kinases and tyrosine phosphatases within mitochondria. The activation of Src kinases is mainly regulated through phosphorylation specific tyrosine residues. Active Src kinases induce some subunits phosphorylation of respiratory complexes, which accelerates the processes of electron transfer and ATP synthesis. Meanwhile, Src kinases inhibits the MPTP opening by regulation the subunits phosphorylation. Tyrosine phosphatases, as dephosphorylation enzymes, reverse the effects of Src kinases on oxidative phosphorylation and MPTP. However, phosphatases such as SHP2 and PTP1B increase the activation of Src kinases through dephosphorylation of inhibiting residues. The effects of Src kinases and tyrosine phosphatases are respectively inhibited by PP2 and vanadate. In the present study, spermine is found to be elevating the activation of Src kinases. Arrows represent activation and ' $\mathrm{T}$ ' symbolizes the inhibition.

and suppresses MPTP opening, which indicates that spermine is involved in mitochondrial protection in isolated spinal cord. Consistently, several studies have demonstrated the beneficial effects of spermine on mitochondria. For example, spermine has been shown to buffer excess mitochondrial $\mathrm{Ca}^{2+}$ to the equivalent level as in the cytosol (26). As a free radical scavenger, spermine can suppress ROS production and retain the reduced sulfhydryl groups at normal levels, exerting an antioxidative effect $(27,28)$. It is also reported that exogenous spermine stabilizes the membrane structures in myocardial ischemia-reperfusion (29). Furthermore, spermine appears to inhibit inflammation in microglial cells (30). However, these conclusions conflict with other hypotheses that spermine is detrimental to cellular metabolism, as it may produce ROS and induce MPTP opening (14). The opposing functions reported for spermine may be caused by the following factors: (i) according to the concentration, spermine may exert dual effects on the mitochondria (31); (ii) depending on the metabolic state, spermine may generate spermine dialdehyde and hydrogen peroxide, which are oxidizing and toxic for cells (32); (iii) as a second messenger, spermine may participate in the signal transduction of various signaling pathways, which intersect with one another and may even exert differential targeting effects. To the best of our knowledge, the definitive mechanism of spermine has not been clarified in spinal cord mitochondria. Therefore, the present study investigated the regulatory effect of spermine on SFKs, which are fundamental signaling components within mitochondria.

Considering that oxidative phosphorylation is the central target of SFKs (33), our first hypothesis deals with the possible effect of spermine in altering mitochondrial respiration, and the results appear to confirm this effect. In the measurement of respiration, state III reflects the maximum 
rate of coupled respiration, and state IV reflects the capacity of proton reflux (10). Therefore, the RCR represents the ability to integrate oxidative phosphorylation. As shown in Fig. 1, the process of oxidative phosphorylation is enhanced by spermine, as demonstrated by the increase of RCR and the oxygen consumption of state III. This effect is not associated with a change in state IV respiration, as the inner membrane remains intact and the $\Delta \Psi_{\mathrm{m}}$ remains at physiological levels (Fig. 2). These results are opposite to that in liver (23). The membrane potential of liver mitochondria is readily decreased by charge neutralization of spermine, while abundant fatty acids within spinal cord mitochondria increase the charge flow across the inner membrane, and ultimately maintain the $\Delta \Psi_{\mathrm{m}}$ in physiological levels (34). Furthermore, PP2, with or without spermine, markedly attenuates the activity of the electron transport chain, illustrating the activating effect of spermine on Src kinases. It should be noted that several subunits of the electron transport chain are regulated by Src kinases in rat brain mitochondria, including the $39 \mathrm{kDa}$ subunit of complex I, flavoprotein of complex II, core protein 2 of complex III, subunit II of complex IV and the $\alpha$ and $\beta$ subunits of complex V (35). These subunits are activated by the phosphorylation of a tyrosine residue. Meanwhile, the tyrosine residues are inactivated by tyrosine phosphatases. In this case, vanadate inhibits dephosphorylation, increasing RCR and the oxygen consumption of state III.

Subsequently, we hypothesized that spermine inhibits MPTP opening by regulating tyrosine kinases. The molecular constituents of the MPTP remain controversial; however, several integral proteins have been confirmed to comprise the MPTP, including VDAC in the mitochondrial outer membrane, AdNT in the inner membrane and cyclophilin $\mathrm{D}$ in the matrix (36). The N-terminal region of VDAC is the site of tyrosine phosphorylation (37), and AdNT is known to be phosphorylated on Y190 and Y194 tyrosine residues (17). Therefore the functions of VDAC and AdNT are conspicuously influenced by tyrosine phosphorylation. As shown in Fig. 3, $1 \mathrm{mM}$ spermine inhibits the opening of MPTPs, the effect of which is partially abolished by PP2, indicating that spermine is involved in regulating the activity of tyrosine kinases. This effect supports the role of spermine in the oxidative respiratory chain. This inhibition on MPTP of spermine is in accordance with that in liver mitochondria as previous reported (24). However, the mechanism of spermine in liver is enhancement the inhibitory effects of ADP rather than the regulation of tyrosine phosphorylation. The degree of opening of the MPTP is influenced by the mitochondrial physiological status. Exogenous $\mathrm{Ca}^{2+}$ is primarily absorbed through a specific uniporter, and this process is dependent on the normal $\Delta \Psi_{\mathrm{m}}$ level (11). As shown in Fig. 2, spermine and other reagents marginally alter the $\Delta \Psi_{\mathrm{m}}$, which maintains the $\Delta \Psi_{\mathrm{m}}$ at physiological levels. The results of $\mathrm{Ca}^{2+}$-induced mitochondrial swelling are dramatically affected by the extent of channel/pore opening in the inner membranes, other than MPTPs. CsA, as a specific inhibitor, is used to suppress the opening of the MPTP by binding to cyclophilin D in the matrix (38). As shown in Fig. 3, CsA effectively counteracted the influx of water from the cytoplasm, illustrating that other channels or pores may be partially opened by $\mathrm{Ca}^{2+}$.
There are 11 SFKs, including Lck, Lyn, c-Src, Srm, Blk, Brk, Fgr, Frk, Fyn, Hck and Yes (39). Among the different members, only Lyn, c-Src, Fgr and Fyn are expressed in the mitochondria (40), and they share a similar domain (Fig. 5A). The activity of Src kinases is primarily regulated by the Y419 residue and the C-terminal Y530 residue (41). The phosphorylated Y530 binds to the SH2 domain, resulting in a closed and inactive conformation, which masks the active Y419 residue (Fig. 5B). In rat brain mitochondria, tyrosine phosphatases, such as SHP-2 and PTP1B, induce the dephosphorylation of Y530 residues (42). The dissociation between the SH2 domain and the Y530 residue exposes the Y419 residue (Fig. 5C). Src kinases are then activated by auto-phosphorylation of Y419 (Fig. 5D). As an inhibitor of phosphatases, vanadate attenuates the dephosphorylation of Y530 and as a result, reduces the level of activated SFKs.

The mechanism of action of spermine within mitochondria is illustrated in Fig. 6 according to the present experimental findings. Spermine activates SFKs, and activated SFKs then induce the phosphorylation of certain subunits of respiratory complexes to accelerate the processes of electron transfer and ATP synthesis. Additionally, Src kinases inhibit MPTP opening by regulating the function of VDAC and AdNT. The phosphatases reverse the effect of SFKs by dephosphorylation of tyrosine residues of the respiratory chain complexes and MPTPs. However, SFKs are activated by phosphatases through dephosphorylation of the Y530 residue. This may be due to negative feedback within the mitochondria. Instead, in liver mitochondria, which have no SFKs, the mitochondrial functions are mainly regulated by the thiol groups and redox state (19). Therefore, the spermine presents diverse mechanisms on distinct organs.

In summary, the results of the present study demonstrate that spermine enhances respiratory processes and reduces the degree of MPTP opening in isolated mitochondria of the spinal cord. The mitochondria-protective properties of spermine probably arise from the regulation on Src kinases, which are the initiating signaling factors of the tyrosine kinase pathway. Further work will evaluate whether secondary SCI is alleviated by spermine in rat models and, if possible, identify the site of action of spermine on SFKs.

\section{References}

1. Hall ED and Springer JE: Neuroprotection and acute spinal cord injury: A reappraisal. NeuroRx 1: 80-100, 2004.

2. Lewen A, Matz P and Chan PH: Free radical pathways in CNS injury. J Neurotrauma 17: 871-890, 2000.

3. Chinopoulos C and Adam-Vizi V: Mitochondrial Ca2+ sequestration and precipitation revisited. FEBS J 277: 3637-3651, 2010

4. Pivovarova NB and Andrews SB: Calcium-dependent mitochondrial function and dysfunction in neurons. FEBS J 277: 3622-3636, 2010.

5. Szabo I and Zoratti M: The mitochondrial megachannel is the permeability transition pore. J Bioenerg Biomembr 24: 111-117, 1992.

6. Zoratti M and Szabó I: The mitochondrial permeability transition. Biochim Biophys Acta 1241: 139-176, 1995.

7. Sesso A, Marques MM, Monteiro MM, Schumacher RI, Colquhoun A, Belizário J, Konno SN, Felix TB, Botelho LA, Santos VZ, et al: Morphology of mitochondrial permeability transition: morphometric volumetry in apoptotic cells. Anat Rec A Discov Mol Cell Evol Biol 281: 1337-1351, 2004.

8. Christofferson DE and Yuan J: Necroptosis as an alternative form of programmed cell death. Curr Opin Cell Biol 22: 263-268, 2010. 
9. Duprez L, Wirawan E, Vanden Berghe T and Vandenabeele P: Major cell death pathways at a glance. Microbes Infect 11: 1050-1062, 2009.

10. McEwen ML, Sullivan PG, Rabchevsky AG and Springer JE: Targeting mitochondrial function for the treatment of acute spinal cord injury. Neurotherapeutics 8: 168-179, 2011.

11. Dalla Via L, Di Noto V, Siliprandi D and Toninello A: Spermine binding to liver mitochondria. Biochim Biophys Acta 1284: 247-252, 1996

12. Siliprandi D, Toninello A and Dalla Via L: Bidirectional transport of spermine in rat liver mitochondria. Biochim Biophys Acta 1102: 62-66, 1992.

13. Pezzato E, Battaglia V,Brunati AM, Agostinelli E and Toninello A $\mathrm{Ca} 2+-$ independent effects of spermine on pyruvate dehydrogenase complex activity in energized rat liver mitochondria incubated in the absence of exogenous $\mathrm{Ca} 2+$ and $\mathrm{Mg} 2+$. Amino Acids 36 : 449-456, 2009

14. Agostinelli E, Tempera G, Molinari A, Battaglia V, Toninello A and Arancia G: The physiological role of biogenic amines redox reactions in mitochondria. New perspectives in cancer therapy. Amino Acids 33: 175-187, 2007.

15. Sava IG, Battaglia V, Rossi CA, Salvi M and Toninello A: Free radical scavenging action of the natural polyamine spermine in rat liver mitochondria. Free Radic Biol Med 41: 1272-1281, 2006.

16. Cesaro L and Salvi M: Mitochondrial tyrosine phosphoproteome: New insights from an up-to-date analysis. Biofactors 36 437-450, 2010

17. Lewandrowski U, Sickmann A, Cesaro L, Brunati AM, Toninello A and Salvi M: Identification of new tyrosine phosphorylated proteins in rat brain mitochondria. FEBS Lett 582: 1104-1110, 2008.

18. Pantic B, Trevisan E, Citta A, Rigobello MP, Marin O, Bernardi P, Salvatori S and Rasola A: Myotonic dystrophy protein kinase (DMPK) prevents ROS-induced cell death by assembling a hexokinase II-Src complex on the mitochondrial surface. Cell Death Dis 4: e858, 2013.

19. Battaglia V, Tibaldi E, Grancara S, Zonta F, Brunati AM, Martinis P, Bragadin M, Grillo MA, Tempera G, Agostinelli E and Toninello A: Effect of peroxides on spermine transport in rat brain and liver mitochondria. Amino Acids 42: 741-749, 2012

20. Battaglia V, Grancara S, Satriano J, Saccoccio S, Agostinelli E and Toninello A: Agmatine prevents the $\mathrm{Ca}(2+)$-dependent induction of permeability transition in rat brain mitochondria. Amino Acids 38: 431-437, 2010.

21. Sullivan PG, Dubé C, Dorenbos K, Steward O and Baram TZ: Mitochondrial uncoupling protein-2 protects the immature brain from excitotoxic neuronal death. Ann Neurol 53: 711-717, 2003.

22. Kristal BS, Park BK and Yu BP: 4-Hydroxyhexenal is a potent inducer of the mitochondrial permeability transition. J Biol Chem 271: 6033-6038, 1996

23. Salvi M, Battaglia V, Mancon M, Colombatto S, Cravanzola C, Calheiros R, Marques MP, Grillo MA and Toninello A: Agmatine is transported into liver mitochondria by a specific electrophoretic mechanism. Biochem J 396: 337-345, 2006

24. Grancara S, Battaglia V, Martinis P, Viceconte N, Agostinelli E, Toninello A and Deana R: Mitochondrial oxidative stress induced by $\mathrm{Ca} 2+$ and monoamines: Different behaviour of liver and brain mitochondria in undergoing permeability transition. Amino Acids 42: 751-759, 2012.

25. Bonaiuto E, Grancara S, Martinis P, Stringaro A, Colone M, Agostinelli E, Macone A, Stevanato R, Vianello F, Toninello A and Di Paolo ML: A novel enzyme with spermine oxidase properties in bovine liver mitochondria: Identification and kinetic characterization. Free Radic Biol Med 81: 88-99, 2015.

26. Salvi M and Toninello A: Effects of polyamines on mitochondrial $\mathrm{Ca}(2+)$ transport. Biochim Biophys Acta 1661: 113-124, 2004.
27. Lapidus RG and Sokolove PM: Spermine inhibition of the permeability transition of isolated rat liver mitochondria: An investigation of mechanism. Arch Biochem Biophys 306: 246-253, 1993

28. Lapidus RG and Sokolove PM: The mitochondrial permeability transition. Interactions of spermine, ADP and inorganic phosphate. J Biol Chem 269: 18931-18936, 1994.

29. Zhao YJ, Xu CQ, Zhang WH, Zhang L, Bian SL, Huang Q, Sun HL, Li QF, Zhang YQ, Tian Y, et al: Role of polyamines in myocardial ischemia/reperfusion injury and their interactions with nitric oxide. Eur J Pharmacol 562: 236-246, 2007.

30. Choi YH and Park HY: Anti-inflammatory effects of spermidine in lipopolysaccharide-stimulated BV2 microglial cells. J Biomed Sci 19: 31, 2012.

31. Wei C, Li HZ, Wang YH, Peng X, Shao HJ, Li HX, Bai SZ, $\mathrm{Lu} \mathrm{XX}, \mathrm{Wu} \mathrm{LY}$, Wang R and Xu CQ: Exogenous spermine inhibits the proliferation of human pulmonary artery smooth muscle cells caused by chemically-induced hypoxia via the suppression of the ERK1/2- and PI3K/AKT-associated pathways. Int J Mol Med 37: 39-46, 2016.

32. Dalla Via L, Mammi S, Uriarte E, Santana L, Lampronti I, Gambari R and Gia O: New furan side tetracyclic allopsoralen derivatives: synthesis and photobiological evaluation. J Med Chem 49: 4317-4326, 2006.

33. Grancara S, Zonta F, Ohkubo S, Brunati AM, Agostinelli E and Toninello A: Pathophysiological implications of mitochondrial oxidative stress mediated by mitochondriotropic agents and polyamines: The role of tyrosine phosphorylation. Amino Acids 47: 869-883, 2015

34. Tahin QS, Blum M and Carafoli E: The fatty acid composition of subcellular membranes of rat liver, heart, and brain: Diet-induced modifications. Eur J Biochem 121: 5-13, 1981.

35. Augereau O, Claverol S, Boudes N, Basurko MJ, Bonneu M, Rossignol R, Mazat JP, Gineste C, Hernandez A, Ivarsson N, Cheng AJ, Naess K, Wibom R, Lesko N, Bruhn H, Wedell A, Freyer C, et al: Cyclophilin D, a target for counteracting skeletal muscle dysfunction in mitochondrial myopathy. Hum Mol Genet 24: 6580-6587, 2015.

36. Crompton M: On the involvement of mitochondrial intermembrane junctional complexes in apoptosis. Curr Med Chem 10 1473-1484, 2003.

37. Distler AM, Kerner J and Hoppel CL: Post-translational modifications of rat liver mitochondrial outer membrane proteins identified by mass spectrometry. Biochim Biophys Acta 1774: 628-636, 2007.

38. Gineste C, Hernandez A, Ivarsson N, et al: Cyclophilin D, a target for counteracting skeletal muscle dysfunction in mitochondrial myopathy. Hum Mol Genet 24: 6580-6587, 2015.

39. Roskoski R, Jr.: Src kinase regulation by phosphorylation and dephosphorylation. Biochem Biophys Res Commun 331: 1-14, 2005.

40. Salvi M, Brunati AM and Toninello A: Tyrosine phosphorylation in mitochondria: a new frontier in mitochondrial signaling. Free Radic Biol Med 38: 1267-1277, 2005.

41. Hebert-Chatelain E: Src kinases are important regulators of mitochondrial functions. Int J Biochem Cell Biol 45: 90-98, 2013.

42. Arachiche A, Augereau O, Decossas M, et al: Localization of PTP-1B, SHP-2, and Src exclusively in rat brain mitochondria and functional consequences. J Biol Chem 283: 24406-24411, 2008 . 